ISSN 1997-5902

\title{
Parasitisme des champs semenciers de cacaoyers par les Loranthaceae dans la localité de Nkoemvone (sud Cameroun)
}

ONDOUA Joseph Marie ${ }^{1}$, DIBONG Siegfried Didier $(2,3,4)^{*}$, TAFFOUO Victor Désiré $(2,4)$, NGOTTA BIYON Jacques Bruno (2)

(1) Département de Botanique et de Physiologie Végétale, Faculté des Sciences, B.P. 63,

Université de Buea, Cameroun

(2) Département de Biologie des Organismes Végétaux, Faculté des Sciences, B.P. 24157, Université de Douala, Cameroun

(3) Département des Sciences Pharmaceutiques, Faculté de Médecine et des Sciences Pharmaceutiques, B.P. 2701, Université de Douala, Cameroun

(4) Institut des Sciences Halieutiques, B.P. 2701, Université de Douala, Cameroun

Auteur de la correspondance : didierdibong@yahoo.fr

Original submitted in on $18^{\text {th }}$ November 2014. Published online at www.m.elewa.org on $30^{\text {th }}$ January 2015 http://dx.doi.org/10.4314/jab.v85i1.1

\section{RESUME}

Objectif : le présent travail consiste à rechercher les méthodes de lutte adéquates contre le parasitisme des Loranthaceae dans les champs semenciers de la SODECAO à Nkoemvone, au Cameroun.

Méthodologie et résultats : l'étude a consisté à dénombrer les différentes espèces de Loranthaceae qui parasitent les champs semenciers, les pieds de cacaoyers infestés et les touffes de parasites rencontrés sur ces arbres, afin de déterminer le taux et l'intensité du parasitisme des cacaoyers et le comportement des différents clones vis-à-vis de l'attaque des Loranthaceae. Les résultats obtenus montrent que Cinq Loranthaceae attaquent les champs semenciers, le niveau d'infestation varie suivant les différents clones, Phragmanthera capitata est la Loranthaceae la plus abondante ; le niveau de parasitisme des cacaoyers est plus important chez les cacaoyers de diamètre moyen.

Conclusion et application des résultats : pour une amélioration des récoltes par les paysans, il est urgent de rechercher les méthodes de lutte appropriées contre le parasitisme des Loranthaceae. En attendant une méthode de lutte efficace, les clones ICS 46 et UPA 146 résistants au parasitisme, peuvent être utilisés pour l'extension des champs semenciers et la distribution aux paysans.

Mots clés : Loranthaceae, semenciers, cacaoyers, clones, parasitisme, Nkoemvone

\section{ABSTRACT}

Objective: The present work consists in looking for appropriate control methods against the parasitism of Loranthaceae in the seed fields of the SODECAO to Nkoemvone, in Cameroon.

Methodology and results: The study consisted in counting the various species of Loranthaceae which live as parasites on the seed fields, on the feet of infested cacao trees and to determine the rate and the intensity of the parasitism of cacao trees and the behaviour of the various clones towards the attack of Loranthaceae. The obtained results show that five Loranthaceae attacked the seed fields, the level of infestation varied according 
to the various clones, Phragmanthera capitata was the most abundant Loranthaceae; the level of parasitism of cacao trees is more important at the cacao trees of average diameter.

Conclusion and application of the results: for an improvement of the harvest by the farmers, it is urgent seek appropriate control methods suited against the parasitism of Loranthaceae. Pending an effective control method, clones ICS 46 and UPA 146 resistant of parasites can be used for the extension of fields and seed distribution to farmers

Key words: Loranthaceae, seed fields, cocoa, clones, parasitism, Nkoemvone

\section{INTRODUCTION}

La relance de la cacaoculture au Cameroun à travers les structures de production des semences tel que la Société de Développement du Cacao (SODECAO) se fonde sur la réhabilitation des plantations existantes et la création de nouvelles plantations (CICC, 2009). L'impact de cette relance est d'acroitre la production à brève échéance par l'État du Cameroun. Les besoins de semences exprimés auprès de la SODECAO par des planteurs demandeurs de matériel végétal imposent à cette société un accroissement de sa capacité de production. Cependant la production cacaoyère dans cette structure fait face à de nombreuses contraintes dont les plus importantes sont liées aux parasites qui causent annuellement des pertes estimées à 40\% de la production mondiale (Eskes et Lanaud, 1997). Parmi ces parasites figurent les Loranthaceae. Les Loranthaceae sont des arbrisseaux épiphytes, chlorophylliens qui vivent en hémiparasites sur les branches d'arbres et d'arbustes, sauvages ou cultivés (Balle, 1982). Ces plantes qui se présentent sous forme de touffes, sont ancrées dans le bois de l'hôte grâce à un suçoir qui permet d'établir des liaisons fonctionnelles avec l'appareil conducteur de l'hôte (Sallé et al., 1998). Le parasite prélève ainsi l'eau, les éléments minéraux et le complément de matière organique dont il a besoin. Le parasitisme par les Loranthaceae est un problème écologique fortement répandu dans le monde (Kuijt, 1969; Polhill and Wiens, 1998). Ces plantes parasites épiphytes chlorophylliennes sont responsables de

\section{MATERIEL ET METHODES}

Le travail repose principalement sur les observations effectuées sur le terrain et sur les enquêtes basées sur des interviews directes.

Site de l'étude : Le site d'étude choisi est la station de production des semences cacaoyères de la SODECAO à dégâts économiques et morphogénétiques variables selon les essences ligneuses parasitées (Sallé et al., 1998). Les Loranthaceae sont largement distribuées dans les régions tropicales: Amérique, Afrique, Australie; elles s'étendent aux régions tempérées avec quelques représentants (Balle, 1982 ; Barlow, 1983). Elles sont regroupées en 950 espèces, reparties en 77 genres dans les régions tempérées. Polhill et Wiens (1998) dénombrent plus de 500 espèces en Afrique et en Arabie tandis que Balle (1982) comptabilise 7 genres et près de 26 espèces au Cameroun. Aujourd'hui, suite à l'évolution des mauvaises conditions climatiques de la sous-région, conséquente à la variation écologique liée à la toponymie, ces hémiparasites sont devenus un fléau réel contre lequel une lutte énergique s'avère indispensable compte tenu de l'ampleur de dégâts. Cependant, Sallé et Aber (1986) signalent que toute lutte acharnée contre les hémiparasites passe nécessairement par une meilleure connaissance de leur écologie et de leur biologie. L'objectif de ce travail est la recherche des méthodes de lutte adéquates contre le parasitisme des Loranthaceae. Les objectifs spécifiques sont: (1) inventorier les clones de cacaoyer et les espèces de Loranthaceae qui les parasitent dans la station de production des champs semenciers, (2) calculer le taux et l'intensité de parasitisme des champs semenciers et déterminer le comportement des clones de cacaoyers vis-à-vis de ce parasitisme.

Nkoemvone au Cameroun. Le choix de ce site est motivé par une fréquence élevée des Loranthaceae dans les champs semenciers et les pertes de rendement de plus en plus importantes. La localité de Nkoemvone située entre $11,10-12,2^{\circ}$ de longitude Ouest et entre $2,4^{\circ}-2,8^{\circ}$ de 

localité de Nkoemvone (sud Cameroun)

latitude Nord à une altitude de $630 \mathrm{~m}$, à $15 \mathrm{~km}$ d'Ebolowa sur la route Ebolowa-Ambam. Ce site est soumis à un climat équatorial humide à quatre saisons : une grande saison sèche (décembre-mars), une petite saison sèche (juin-août), une grande saison des pluies (septembrenovembre) et une petite saison des pluies (avril - mai). Ces précipitations atteignent $1755 \mathrm{~mm}$ et la température moyenne est de $25,5^{\circ} \mathrm{C}$. (Fines et al., 2001). Mise en place à partir de 1960 avec environ 27 champs semenciers numéroté de 1 à 27 sur une superficie de près de 100 ha avec 1000 pieds de cacaoyers à l'hectare, aujourd'hui il n'en reste qu'environ 11 sur une superficie de près de 50 ha. Chaque champ est composé de deux clones. Chaque clone s'écrit en majuscule avec les initiales de la localité d'origine suivi d'un numéro d'ordre. Les clones sélectionnés dans les collections d'origine locale de la Station de Nkoemvone sont désignés par trois lettres SNK, c'est-à-dire, sélection de Nkoemvone, suivies d'un numéro d'ordre (Exemple, SNK 13). Les clones repérés dans les descendances de cacaoyers haut-amazoniens sont désignes par 3 lettres UPA (Upper Amazon), suivies d'un numéro d'ordre (UPA 143). La nomenclature des clones sélectionnés par I'Imperial College de Trinidad a été conservée, clones ICS (ICS 40).

Échantillonnage dans les champs semenciers : Sur les 11 champs réhabilités et actuellement en exploitation dans la station de Nkoemvone, 10 ont été retenus dans le cadre de cette étude pour une superficie de 35,11 ha. Ces 10 champs semenciers retenus ont été parcourus et

\section{RESULTATS}

Caractérisation de l'infestation

Inventaire des Loranthaceae sur les cacaoyers : Cinq espèces de Loranthaceae ont été inventoriées sur les cacaoyers. II s'agit de Globimetula dinklagei (Engl.) tous les pieds de cacaoyer minutieusement explorés. Les pieds de cacaoyers de chaque clone ont été comptabilisés ainsi que les pieds de cacaoyers parasités. Le nombre de touffes de chaque parasite rencontré a été noté. Les échantillons ont été prélevés à l'aide d'un scalpel, emballés dans des papiers journaux puis mis dans une presse en bois. Une partie de l'identification s'est faite sur place à l'aide des différentes clés d'identification et l'autre complétée au Laboratoire de Biologie et Physiologie Végétales de la Faculté des Sciences de l'Université de Douala. Le diamètre de chaque pied de cacaoyer parasité a été noté. Les touffes de parasites présents sur chaque pied de cacaoyer ont été comptabilisées. Ces données ont permis de déterminer :

- le taux de parasitisme qui est le pourcentage des pieds de cacaoyer parasités dans un champ donné ;

- l'intensité de parasitisme qui exprime l'ampleur de l'infestation sur les pieds de cacaoyer parasités dans un champ ; elle est exprimée, par le nombre moyen de touffes de Loranthaceae observées par clone et par champ.

Les travaux se sont déroulés d'octobre à décembre 2012, période pendant laquelle les Loranthaceae ont été en fleurs et faciles à identifier. Les données de terrain ont été compilées et traitées dans le logiciel Excel du pack MICROSOFT OFFICE 2007. Le logiciel GRAPHPAD Prisme 5 a été utilisé pour l'évaluation des corrélations entre les différents paramètres d'étude relevés et les différents tests de significativité.

Danser, Phragmanthera capitata (Spreng.) Balle, Phragmanthera nigritana (Hook.f. ex Benth.) Balle, Tapinanthus ogowensis (Engl.) Danser et Tapinanthus preussii (Engl.) Tiegh (Fig 1 a, b, c, d et e). 
Ondoua et al.. J. Appl. Biosci. Parasitisme des champs semenciers de cacaoyers par les loranthaceae dans la localité de Nkoemvone (sud Cameroun)
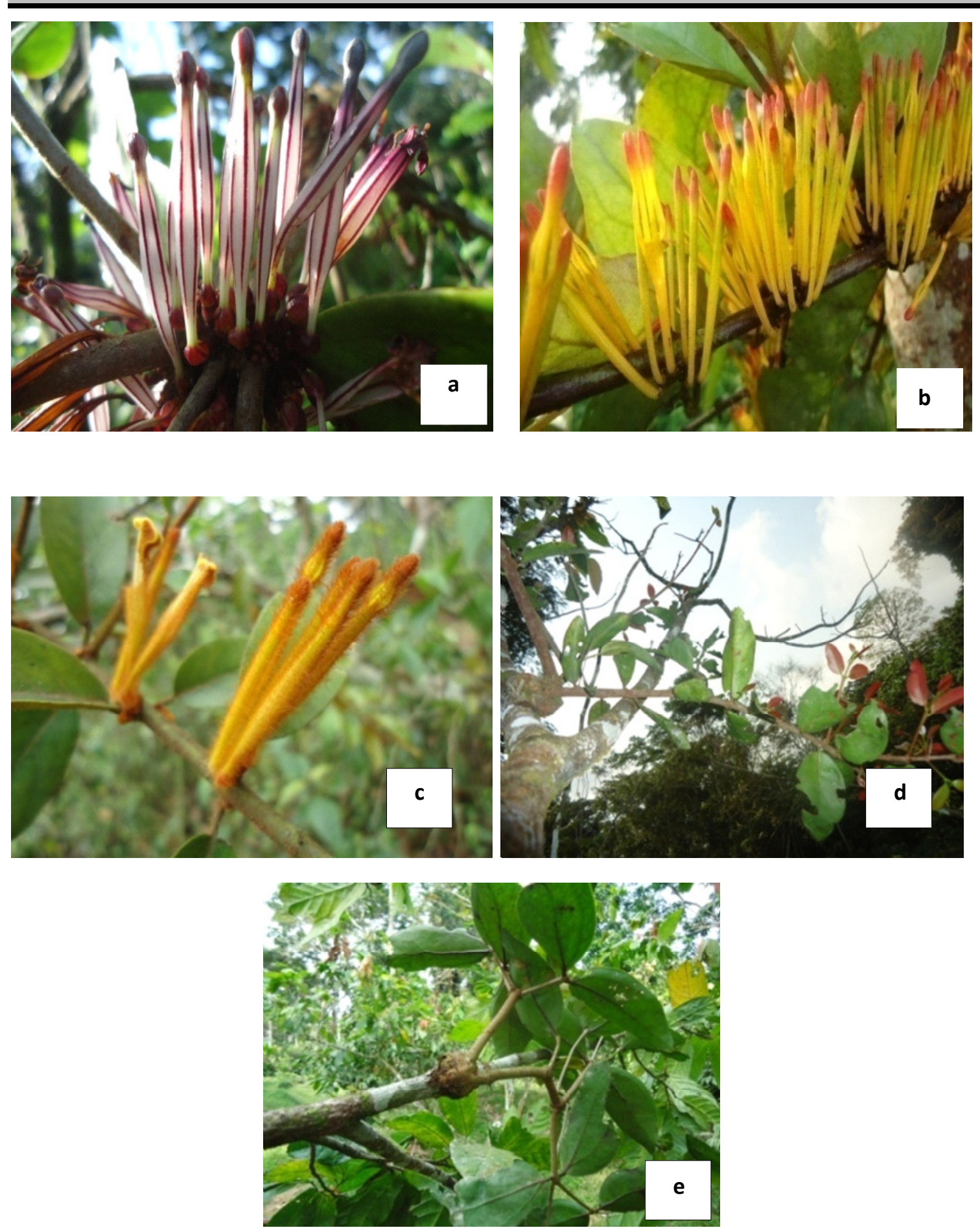

Fig.1. Loranthaceae inventoriées : (a) Globimetula dinklagei (Engl.) Danser ; (b) Phragmanthera capitata (Spreng.); (c) Phragmanthera nigritana (Hook.f. ex Benth.) Balle; (d) Tapinanthus ogowensis (Engl.) Danser et (e) Tapinanthus preussii (Engl.) Tiegh.

Le nombre de touffes de Loranthaceae varie d'une espèce à l'autre (Tableau 1). L'abondance des
Loranthaceae sur les pieds de cacaoyer dans les différents champs se présente de la manière suivante : 
Ondoua et al.. J. Appl. Biosci. Parasitisme des champs semenciers de cacaoyers par les loranthaceae dans la localité de Nkoemvone (sud Cameroun)

Globimetula dinklagei (Engl.) Danser 0,07 \%, Phragmanthera nigritana (Hook.f. ex Benth.) Balle 5,5\%, Tapinanthus ogowensis (Engl.) Danser 0,07 \%, et Phragmanthera capitata (Spreng.) Ballé 94,14\%.

Tapinanthus preussii (Engl.) Tiegh. 0,22 \%,

Tableau 1 : Inventaire des Loranthaceae dans les champs semenciers.

\begin{tabular}{|l|l|l|}
\hline Espèces de Loranthceae & Nombre de touffes & Taux de parasitisme (\%) \\
\hline Globimetula dinklagei & 1 & 0,07 \\
\hline Phragmanthera capitata & 1302 & 94,14 \\
\hline Phragmanthera nigritana & 76 & 5,5 \\
\hline Tapinanthus ogowensis & 1 & 0,07 \\
\hline Tapinanthus preussii & 3 & 0,22 \\
\hline Totaux & 1383 & 100 \\
\hline
\end{tabular}

Taux de parasitisme : Dans les 10 champs, 7605 pieds de cacaoyer ont été comptabilisés dont 794 parasités (Tableau 2). De même, 1383 touffes de Loranthaceae ont été identifiées (Fig. 2). Le taux de parasitisme de chaque champ montre que les champs $01,02,19,20,22$ et 24 sont les plus parasités avec plus de $15 \%$ du taux de parasitisme. Les taux de parasitisme respectifs de ces champs sont de $17,48 \% ; 16,48 \% ; 15,40 \% ; 15,91 \%$ et $17,84 \%$, tandis que les champs $04,05,16$, et 21 sont les moins parasités avec un taux de parasitisme inférieur à $6 \%$. Le champ 01 présente le plus grand nombre de touffes soit 429 , alors que les champs 05 et 21 sont les moins parasités avec 04 touffes chacun. L'analyse de variance indique une différence significative entre les taux d'infestation des champs ( $P$ value $<0,001)$. Le champ 22 est le plus parasité tandis que le champ 5 est le moins parasité.
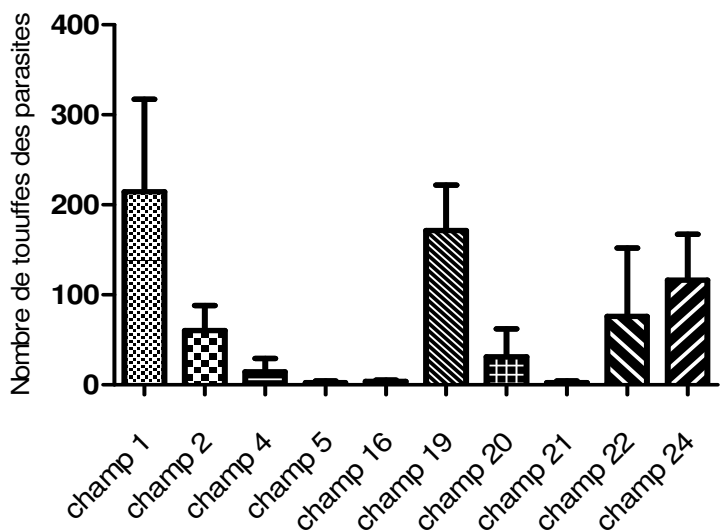

Fig. 2 : Nombre de touffes de Loranthaceae en fonction des champs semenciers.

Tableau 2 : Inventaire des cacaoyers parasités dans les champs biclonaux de Nkoemvone.

\begin{tabular}{|c|c|c|c|c|c|c|c|}
\hline No du champ & Clones & Superficie (ha) & NTPC & NTPCP & NTPCC & NTP & TPC (\%) \\
\hline \multirow[t]{2}{*}{01} & UPA 143 & \multirow[t]{2}{*}{1,5} & \multirow[t]{2}{*}{1436} & 177 & 523 & \multirow[t]{2}{*}{429} & \multirow[t]{2}{*}{17,48} \\
\hline & SNK 64 & & & 74 & 913 & & \\
\hline \multirow[t]{2}{*}{02} & SNK 13 & \multirow[t]{2}{*}{1,5} & \multirow[t]{2}{*}{437} & 7 & 118 & \multirow[t]{2}{*}{120} & \multirow[t]{2}{*}{16,48} \\
\hline & ICS 95 & & & 65 & 319 & & \\
\hline \multirow[t]{2}{*}{04} & ICS 46 & \multirow[t]{2}{*}{03} & \multirow[t]{2}{*}{464} & 00 & 386 & \multirow[t]{2}{*}{29} & \multirow[t]{2}{*}{5,39} \\
\hline & T60/887 & & & 25 & 78 & & \\
\hline \multirow[t]{2}{*}{05} & SNK 16 & \multirow[t]{2}{*}{5} & \multirow[t]{2}{*}{665} & 04 & 573 & \multirow[t]{2}{*}{04} & \multirow[t]{2}{*}{0,60} \\
\hline & UPA 146 & & & 00 & 92 & & \\
\hline \multirow[t]{2}{*}{16} & UPA 134 & \multirow[t]{2}{*}{3,5} & \multirow[t]{2}{*}{254} & 02 & 191 & \multirow[t]{2}{*}{07} & \multirow[t]{2}{*}{2,36} \\
\hline & ICS 40 & & & 04 & 63 & & \\
\hline
\end{tabular}


Ondoua et al.. J. Appl. Biosci. Parasitisme des champs semenciers de cacaoyers par les loranthaceae dans la localité de Nkoemvone (sud Cameroun)

\begin{tabular}{|c|c|c|c|c|c|c|c|}
\hline \multirow[t]{2}{*}{19} & INC 67 & \multirow[t]{2}{*}{3,5} & \multirow[t]{2}{*}{1247} & 119 & 375 & \multirow[t]{2}{*}{343} & \multirow[t]{2}{*}{15,40} \\
\hline & SNK 10 & & & 73 & 872 & & \\
\hline \multirow[t]{2}{*}{20} & T79/501 & \multirow[t]{2}{*}{3,5} & \multirow[t]{2}{*}{176} & 28 & 123 & \multirow[t]{2}{*}{62} & \multirow[t]{2}{*}{15,91} \\
\hline & SNK 13 & & & 00 & 53 & & \\
\hline \multirow[t]{2}{*}{21} & T79/467 & \multirow[t]{2}{*}{3,5} & \multirow[t]{2}{*}{392} & 04 & 314 & \multirow[t]{2}{*}{04} & \multirow[t]{2}{*}{1,02} \\
\hline & SNK 13 & & & 00 & 78 & & \\
\hline \multirow[t]{2}{*}{22} & T79/501 & \multirow[t]{2}{*}{2,91} & \multirow[t]{2}{*}{370} & 66 & 203 & \multirow[t]{2}{*}{152} & \multirow[t]{2}{*}{17,84} \\
\hline & SNK 64 & & & 00 & 167 & & \\
\hline \multirow[t]{2}{*}{24} & SNK 16 & \multirow[t]{2}{*}{7,2} & \multirow[t]{2}{*}{2164} & 103 & 1418 & \multirow[t]{2}{*}{233} & \multirow[t]{2}{*}{6,75} \\
\hline & T60/1174 & & & 43 & 746 & & \\
\hline \multicolumn{2}{|c|}{ TOTAUX } & 35,11 & 7605 & 794 & 7605 & 1383 & 10,44 \\
\hline
\end{tabular}

NTPC : Nombre Total de Pieds de Cacaoyer ; NTPCP : Nombre Total de Pieds de Cacaoyer Parasités ; NTP : Nombre Total de Touffes de Parasite ; TPC : Taux de Parasitisme par Champ. NTPCC : Nombre Total de Pieds de Cacaoyer par Clone.

Le taux de parasitisme varie d'un clone à un autre (Fig. 3). Le clone ICS 40 est parasité à $6,35 \%$; ICS 95 à $20,38 \%$; IMC 67 à $31,71 \%$; SNK 10 à $8,37 \%$; SNK 13 à $2,81 \% ;$ SNK 16 à $5,37 \%$; SNK 64 à $6,85 \% ;$ T60/887 à $32,05 \% ;$ T60/1174 à $5,76 \%$, T79/467 à $1,27 \%$; T79/501 à 28,83\%; UPA 134 à 1,05\% et UPA 143 à
$33,84 \%$. L'analyse de variance indique une différence significative $(p<0,05)$ entre les clones. Cette analyse montre également que le test n'est pas significatif entre les clones ICS 46 et UPA 146, les clones T60/1174 et UPA 143. Le clone UPA est le plus parasité alors que les clones ICS 40 et UPA 146 ne le sont pas du tout.

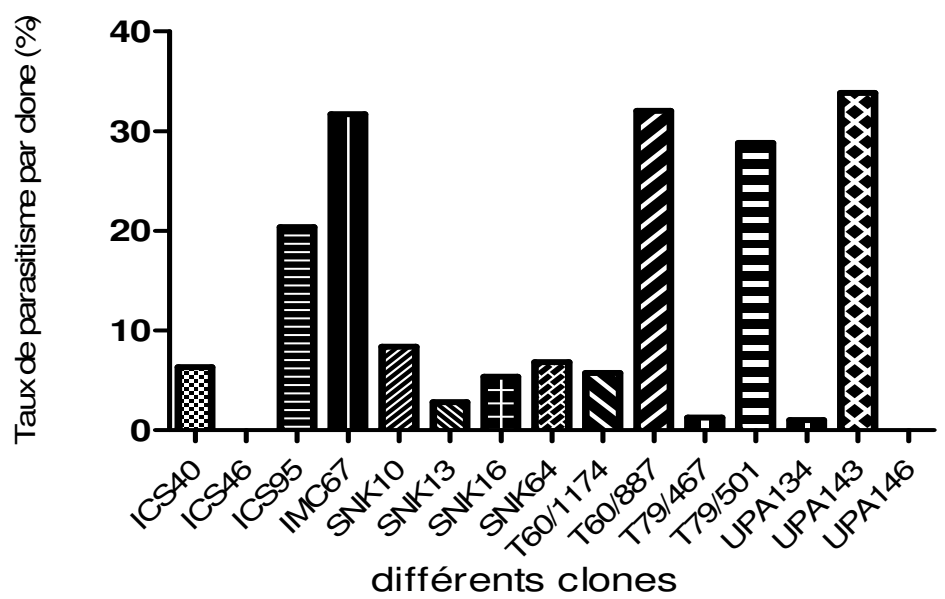

Fig. 3 : Taux de parasitisme en fonction des clones.

Le taux de parasitisme des pieds de cacaoyer varie en fonction des classes de diamètre. Le taux des pieds parasités de la classe $[0 ; 5$ [ est de $3,53 \%$; celui de la classe $[5 ; 10$ [ de $54,53 \%$; celui de la classe [ $10 ; 15$ [ de $32,49 \%$ et celui de la classe [15; 20[ de 6,06\%.
L'analyse de variance, portant sur le taux de parasitisme en fonction des classes de diamètre montre qu'il y a une différence significative entre les pieds de cacaoyer de diamètre $[0 ; 5$ [ et les pieds de cacaoyer de diamètre [ 5 ; 10[ $(P<0,05)$. 


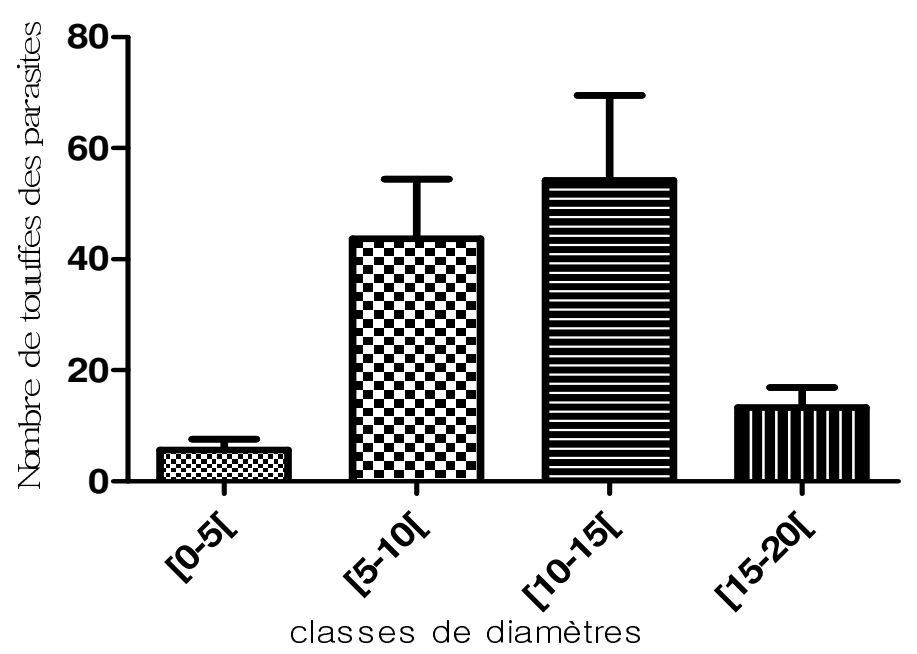

Fig. 4 : Parasitisme des pieds de cacaoyer en fonction des classes de diamètre.

Comportement des clones associés vis-à-vis des Loranthaceae : Le clone SNK 13 est parasité lorsqu'il est associé au clone ICS 95 dans le champ 2 (Tableau 3). Par contre SNK 13 associé avec T79/501 et T79/467 respectivement dans les champs 20 et 21 n'est pas parasité. Le clone SNK 13 en association avec ICS 95 est parasité uniquement chez les individus dont le diamètre varie entre 15 et $20 \mathrm{~cm}$. Le clone SNK 16 est parasité quelque soit le clone qui lui est associé. II en de même avec le clone $T 79 / 501$. Les classes de diamètre $[5 ; 10[$ et $[10 ; 15$ [ sont les plus parasités avec respectivement 657 et 540 pieds de cacaoyer parasités.

Tableau 3 : Comportement de certains clones en association avec d'autres.

\begin{tabular}{|c|c|c|c|c|c|c|}
\hline \multirow[b]{2}{*}{ Champs } & \multirow[b]{2}{*}{ Clones } & \multicolumn{4}{|c|}{ Classes de diamètre } & \multirow[t]{2}{*}{ NPCP } \\
\hline & & {$[0 ; 5[$} & {$[5 ; 10[$} & {$[10 ; 15[$} & {$[15 ; 20]$} & \\
\hline \multirow[b]{2}{*}{01} & UPA143 & 5 & 148 & 143 & 21 & 317 \\
\hline & SNK16 & 2 & 54 & 42 & 14 & 112 \\
\hline \multirow[b]{2}{*}{02} & SNK 13 & 0 & 0 & 0 & 32 & 32 \\
\hline & ICS 95 & 5 & 65 & 18 & 0 & 88 \\
\hline \multirow[t]{2}{*}{04} & ICS 46 & 0 & 0 & 0 & 0 & 00 \\
\hline & T60/887 & 0 & 23 & 5 & 1 & 29 \\
\hline \multirow[t]{2}{*}{05} & SNK 16 & 0 & 3 & 1 & 0 & 04 \\
\hline & UPA146 & 0 & 0 & 0 & 0 & 00 \\
\hline \multirow[t]{2}{*}{16} & UPA134 & 0 & 2 & 0 & 0 & 02 \\
\hline & ICS 40 & 0 & 1 & 4 & 0 & 05 \\
\hline \multirow[t]{2}{*}{19} & IMC 67 & 8 & 83 & 95 & 34 & 220 \\
\hline & SNK10 & 1 & 68 & 43 & 11 & 123 \\
\hline \multirow[t]{2}{*}{20} & $\mathrm{~T} 79 / 501$ & 1 & 18 & 39 & 4 & 62 \\
\hline & SNK 13 & 0 & 0 & 0 & 0 & 00 \\
\hline \multirow[t]{2}{*}{21} & T79/467 & 0 & 3 & 0 & 1 & 04 \\
\hline & SNK 13 & 0 & 0 & 0 & 0 & 00 \\
\hline \multirow[t]{2}{*}{22} & $\mathrm{~T} 79 / 501$ & 1 & 56 & 67 & 28 & 152 \\
\hline & SNK 64 & 0 & 0 & 0 & 0 & 00 \\
\hline \multirow[t]{2}{*}{24} & SNK 16 & 5 & 82 & 74 & 6 & 167 \\
\hline & T60/1174 & 4 & 51 & 9 & 2 & 66 \\
\hline Totaux & & 32 & 657 & 540 & 154 & 1383 \\
\hline
\end{tabular}



localité de Nkoemvone (sud Cameroun)

NPCP : Nombre de pieds de cacaoyer parasités

Intensité de parasitisme : L'intensité de parasitisme des pieds de cacaoyer varie suivant les différents clones (Tableau 4). Cette intensité varie entre 4,57 et 0 touffes de Loranthaceae/pied de cacaoyer parasité. Elle est de 4,57 touffes chez le clone SNK 13 et 0 touffe chez les clones ICS 46 et UPA 146. L'analyse statistique montre trois groupes différents. Le groupe le plus parasité est constitué uniquement du clone SNK 13. Celui du groupe moyennement parasité est constitué des clones ICS 40, ICS 95, IMC 67, SNK 10, SNK 16, SNK 64, T60/887, T60/1174, T79/467, T79/501, UPA 134 et UPA 143. Enfin, celui du groupe non parasité constitué des clones ICS 46 et UPA 146. Les pieds de cacaoyer de classes de diamètre [0-5[ ont une intensité moyenne d'infestation de 1,14 touffes de Loranthaceae/pied de cacaoyer parasité, ceux de la classe de diamètre $[5 ; 10$ ont 1,52 touffes de Loranthaceae/pied de cacaoyer parasité, ceux de la classe [10;15[ ont 1,89 touffes de Loranthaceae/pied de cacaoyer parasité et ceux de la classe de diamètre [1520] ont 3,21 touffes de Loranthaceae/pied de cacaoyer parasité. L'analyse statistique montre deux groupes différents. Le groupe le plus infesté est constitué uniquement des cacaoyers dont le diamètre est supérieur à $15 \mathrm{~cm}$ et le groupe le moins infesté donc les classes de diamètre sont inférieures à $15 \mathrm{~cm}$

Tableau 4 : Inventaire des clones de cacaoyers parasités et du nombre de touffes de Loranthaceae.

\begin{tabular}{|c|c|c|c|c|c|}
\hline Clones & NTPC & NTPCP & NTP & TPC & IP (NTP/NTPCP) \\
\hline ICS 40 & 63 & 04 & 05 & 6,35 & 1,25 \\
\hline ICS 46 & 386 & 00 & 00 & 00 & 00 \\
\hline ICS 95 & 319 & 65 & 88 & 20,38 & 1,35 \\
\hline IMC 67 & 375 & 119 & 220 & 31,71 & 1,85 \\
\hline SNK 10 & 872 & 73 & 123 & 8,37 & 1,68 \\
\hline SNK 13 & 249 & 7 & 32 & 2,81 & 4,57 \\
\hline SNK 16 & 1991 & 107 & 171 & 5,37 & 1,6 \\
\hline SNK 64 & 1080 & 74 & 112 & 6,85 & 1,5 \\
\hline T60/887 & 78 & 25 & 29 & 32,05 & 1,16 \\
\hline T60/1174 & 746 & 43 & 66 & 5,76 & 1,53 \\
\hline T79/467 & 314 & 04 & 04 & 1,27 & 1 \\
\hline T79/501 & 326 & 94 & 214 & 28,83 & 2,28 \\
\hline UPA 134 & 191 & 02 & 02 & 1,05 & 1 \\
\hline UPA 143 & 523 & 177 & 317 & 33,84 & 1,79 \\
\hline UPA 146 & 92 & 00 & 00 & 00 & 00 \\
\hline TOTAUX & 7605 & 794 & 1383 & - & - \\
\hline
\end{tabular}

NTPC : Nombre Total de Pieds de Cacaoyer ; NTPCP : Nombre Total de Pieds de Cacaoyer Parasités ; NTP : Nombre de Touffes de Parasite ; TPC : Taux de Parasitisme par Champ ; IPC : Intensité du parasite par clone 


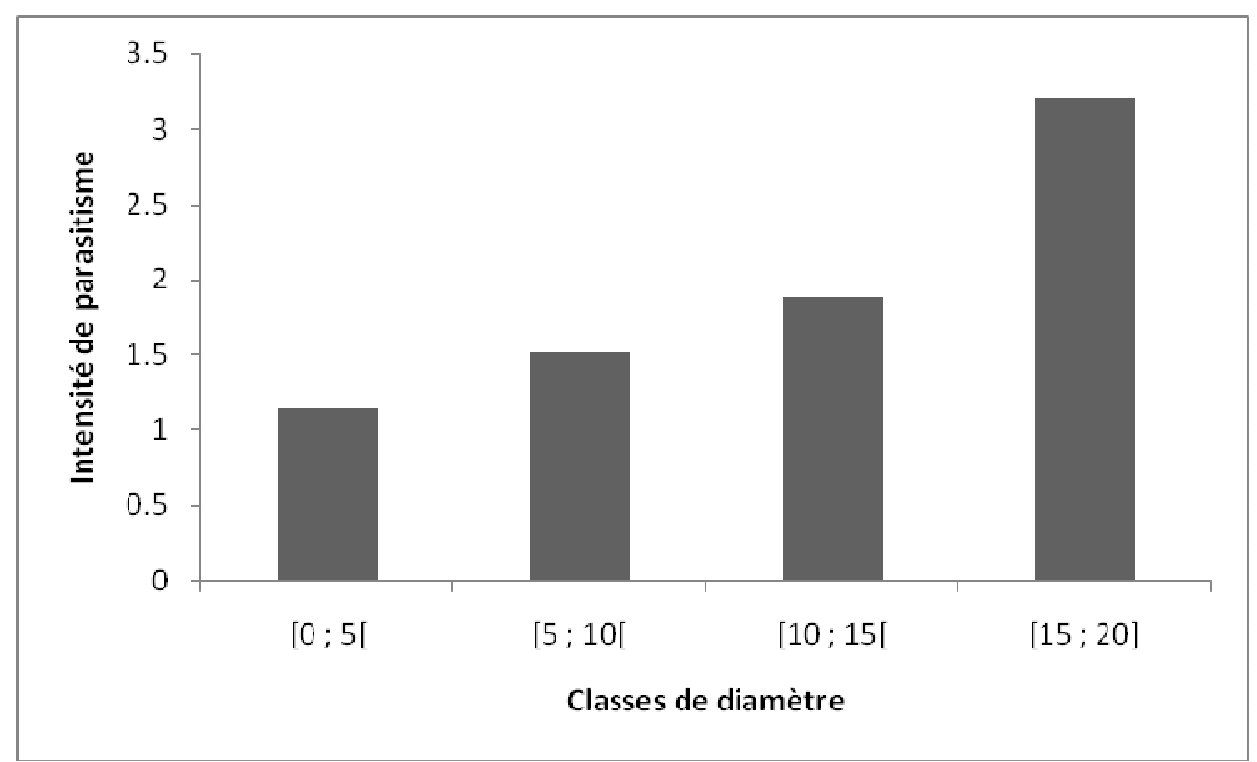

Fig. 5 : Intensité de parasitisme en fonction des classes de diamètre.

\section{DISCUSSION}

Espèces de Loranthaceae rencontrées : Cinq espèces de Loranthaceae ont été inventoriées. II s'agit de Globimetula dinklagei (Engl.) Danser, Phragmanthera capitata (Spreng.) Balle, Phragmanthera nigritana (Hook.f. ex Benth.) Balle, Tapinanthus ogowensis (Engl.) Danser et Tapinanthus preussii (Engl.) Tiegh. Cette diversité est élevée par rapport à celle obtenue dans les plantations d'hévéa de Nyétté, où seule Phragmanthera capitata était présente (Dibong et al., 2009). Phragmanthera capitata est une Loranthaceae ubiquiste de la région du sud Cameroun qui parasite la plupart des arbres cultivés (agrumes, avocatiers, cacaoyers, caféiers, goyaviers...) ou spontanés dont les fruits sont commercialisés et exportés (Dibong et al., 2009). Engone Obiang et Sallé (2006) signalent la présence de la même espèce dans les plantations d'hévéa au Gabon, où elle est plus abondante et associée à d'autres Loranthaceae compagnes. Elle a été associée également à d'autres Loranthaceae compagnes dans les plantations d'hévéa du Sud-Est de la Côte d'Ivoire (Amon et al., 2010) . II résulte un détournement trophique de l'eau et des éléments minéraux, responsable d'importantes pertes économiques (Dibong et al., 2009). A l'origine, toutes les Loranthaceae citées existent et se trouvent dans la forêt et certaines s'adaptent aux milieux modifiés par l'homme et aux espèces ligneuses hôtes cultivées ou spontanées (Dibong et al., 2008). Le passage de la forêt aux milieux modifiés par l'homme s'est opéré sur place via les disséminateurs (Dibong et al., 2009). Au départ, certains individus de Phragmanthera capitata ont pu s'adapter à des espèces hôtes nouvelles, mais qui leur assurent une nutrition facile et abondante. C'est le cas des champs semenciers de Nkoemvone. Une fois installée, elle favorise l'arrivée d'autres espèces de Loranthaceae et certaines associations de clone sont plus favorisées que d'autres. Tels sont les cas des clones UPA 143 et SNK 64, SNK 13 et ICS 96, IMC 67 et SNK 10, T79/501 et SNK 64 qui ont un taux parasitisme supérieur à $15 \%$. Par contre, l'association SNK 16 et UPA 146, T79/467 et SNK 13, UPA 134 et ICS 40 ont un taux de parasitisme inférieur à $3 \%$.

Taux de parasitisme des pieds de cacaoyer : Le taux moyen de parasitisme $(10,44 \%)$ obtenu sur les cacaoyers dans les champs semenciers est moins important que celui observé par Soro et al. (2010) soit $14,10 \%$ et sensiblement égal à $10,40 \%$, valeur obtenue par Koffi (2004) sur des hévéas au Sud de la Côte d'Ivoire. Ce taux est également inférieur à celui de Dibong et al. (2009) qui oscillait entre $17,53 \%$ et $35,05 \%$ dans les plantations d'hévéa de la région Littorale du Cameroun. Ce taux de parasitisme varie suivant les clones. Certains s'avèrent résistants au parasitisme des Loranthaceae; c'est le cas des clones ICS 46 et UPA 146.

Niveau de parasitisme des pieds de cacaoyer selon leur classe de diamètre : Le taux de parasitisme en fonction des classes de diamètre des pieds de cacaoyer parasités montre que les Loranthaceae attaquent le plus les individus de diamètre moyen et que le parasitisme se poursuivra dans le temps et se répandra dans toute la 
région. Pour une amélioration des récoltes par les paysans, il est donc urgent de rechercher les méthodes de lutte appropriées contre le parasitisme des Loranthaceae. Cette étude a montré une différence

\section{CONCLUSION}

Cinq Loranthaceae parasitent les cacaoyers dans les champs semenciers de Nkoemvone. Phragmanthera capitata (Spreng.) Balle est apparue comme la plus abondante dans les champs. Cette étude a montré que tous les clones ne sont pas parasités. Certains clones sont parasités à des degrés différentes en fonction de leur association à d'autres clones Les pieds de cacaoyer plus parasités sont situés dans les classes de diamètre

\section{REFERENCES BIBLIOGRAPHIQUES}

Amon ADE, Soro D, N'Guessan K, Traoré D, 2010. Les Loranthaceae: plantes vasculaires parasites des arbres et arbustes, au Sud-Est de la Côte d'Ivoire. J.Appl. Biosci. 25 : 1565-1572.

Balle S, 1982. Loranthaceae. In Flore du Cameroun (Vol. 23), Satabié B, Leroy JF (eds). Délégation Générale à la Recherche Scientifique et Technique : Yaoundé, Cameroun. 82p.

Barlow BA 1983. Biogeography of Loranthaceae and Viscaceae. The Biology of Mistletoes (Calder M, Bernhardt P), Academic Press, Sydney. 68$131 \mathrm{pp}$.

CICC, 2009. Évaluation intégrée de la libéralisation du secteur cacao sur la biodiversité en République du Cameroun

Dibong SD, Din N, Priso RJ, Taffouo VD, Fankem H, Sallé G, Amougou A, 2008. Parasitism of host trees by the Loranthaceae in region of Douala (Cameroon). African Journal of Environmental Science and Technology 2 (11): 371- 378.

Dibong SD, Din N, Engone Obiang NL, , Priso RJ, Taffouo VD, Fankem H, Sallé G, Amougou A, 2009. Niveau d'infestation des arbres fruitiers des groupements végétaux par Phragmanthera capitata (Sprengel) S. Balle (Loranthaceae) dans la région littorale du Cameroun. Int. J.Biol. Chem. Sci. 3 (2): 347-354.

Dibong SD, Mony R, Ladoh CF, Boussim IJ, Amougou A, 2011. Parasitism evolution of Loranthaceae in the Ndogbong Chiefdom's orchard (Douala,Cameroon). International journal of significative entre le parasitisme des pieds de cacaoyer, selon les quatre classes de diamètre définies dans les champs semenciers.

[5 ; 10[et [10 ; 15[. Pour une amélioration des récoltes par les paysans il est donc urgent de rechercher les méthodes de lutte appropriées contre le parasitisme des Loranthaceae. En attendant une méthode de lutte efficace, les clones ICS 46 et UPA 146 résistants au parasitisme peuvent être utilisés pour l'extension des champs semenciers et la distribution aux paysans.

Plant, Animal and Environmental Sciences 3 (1): 261-269.

Engone Obiang NL, Sallé G, 2006. Faut-il éradiquer Phragmanthera capitata, parasites des hévéas en Afrique ?. C. R. Biologies 329 : 185-195.

Eskes A \& Lanaud C, 1997. Le cacaoyer. In: L'amélioration des Plantes Tropicales, CIRAD, Monpellier, France. 141-170 pp.

Fines JP, Ngibaot F, Ngono G, 2001. A conceptual forest management plan for a medium size forest in southern Cameroon. Tropenbos Cameroon. 185 p.

Koffi AA, 2004. Évaluation de l'incidence des Loranthaceae sur la productivité de Hevea brasilensis (Kunth) Müll. Arg. à Anguédedou (Sud de la Côte d'Ivoire). Mémoire de D.E.A.de Botanique, Université de Cocody, Abidjan. $52 \mathrm{p}$.

Kuijt JL, 1969. The Biology of Parasitic Flowering Plants, University of California Press, Berkeley, Los Angeles. $246 \mathrm{p}$.

Polhill R \& Wiens D, 1998. Mistletoes of Africa, The Royal Botanic, Kew: $370 \mathrm{p}$.

Sallé G \& Aber M, 1986. Les Phanérogames parasites : biologie et stratégies de lutte. Bull. Soc. Bot. Fr. 133: 235-263.

Sallé G, Tuquet C, Raynal-Roques A, 1998. Biologie des phanérogames parasites. C. R. Soc. Biol., 192: 9-36.

Soro K, Soro D, N'Guessam K, Gnahoua GM, Traoré D, 2010. Journal of Animal \& Plant Sciences 6 (1): 597-604. 